\title{
Decay Processes in Cationic Alkali Metals in Microsolvated Clusters: A Complex Absorbing Potential Based Equation-of-Motion Coupled Cluster Investigations
}

\author{
Ravi Kumar ${ }^{\dagger \ddagger}$, Aryya Ghoshף, and Nayana Vaval ${ }^{\ddagger}$ \\ ${ }^{\dagger}$ Academy of Scientific and Innovative Research, CSIR-Human Resource \\ Development Center (CSIR-HRDC) Campus, Postal Staff College Area, Ghaziabad, \\ Uttar Pradesh, 201002, India,

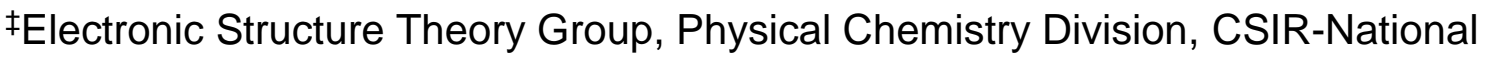 \\ Chemical Laboratory, Pune, 411008, India, and \\ IDepartment of Chemistry, Ashoka University, Sonipat, Haryana,131029 India \\ E-mail: np.vaval@ncl.res.in
}

\section{CONTENTS}

1. Optimized coordinates of different molecules in Z-matrix format 1

2. Modified basis set exponents

OPTIMIZED COORDINATES OF DIFFERENT MOLECULES IN ZMATRIX FORMAT USING GAUSSIANO9 (EXCEPT GEOMETRY 1 \& 3).
$\mathbf{L i}^{+}-\mathbf{H}_{2} \mathrm{O}$
@ CCSD/aug-cc-pVDZ (using Cfour package)

$\begin{array}{cccccccc}\mathrm{O} & 1 & 1.86703 & & & & \\ \mathrm{H} & 2 & 0.96895 & 1 & 127.8659 & & \\ \mathrm{H} & 2 & 0.96895 & 3 & 104.2682 & 1 & 180.0000\end{array}$

$\mathbf{L i}^{+}-\mathbf{N H}_{3}$

@ MP2/ aug-cc-pVTZ 


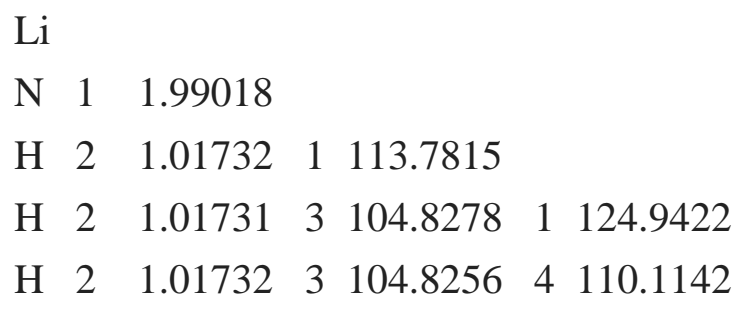

$\mathrm{Na}^{+}-\mathrm{H}_{2} \mathrm{O}$

@ CCSD/aug-cc-pVTZ (using Cfour package)

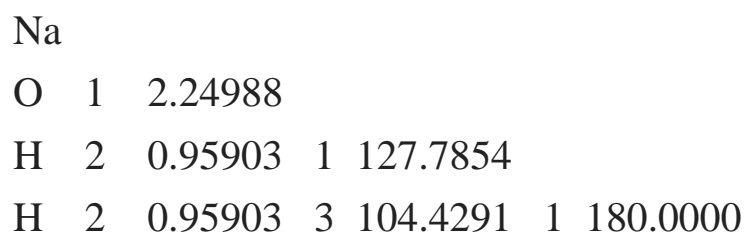

@ B3LYP/6-311++g(3d,2p)

$\mathrm{K}$

$\begin{array}{lll}\text { O } & 1 & 2.62194\end{array}$

$\begin{array}{lllllll}\mathrm{H} & 2 & 0.96371 & 1 & 127.6687\end{array}$

H $2 \begin{array}{llllll}2 & 0.96371 & 3 & 104.6624 & 1 & 180.0000\end{array}$
$\mathbf{L i}^{+}-\left(\mathbf{H}_{2} \mathbf{O}\right)_{2}$
(a B3LYP/6-311++g(3d,2p)

$\mathrm{Li}$

$\begin{array}{lll}\mathrm{O} & 1 & 1.86037\end{array}$

$\begin{array}{lllll}\text { H } & 2 & 0.96434 & 1 & 127.0579\end{array}$

$\begin{array}{lllllll}\mathrm{H} & 2 & 0.96434 & 3 & 105.8841 & 1 & 180.0000\end{array}$

$\begin{array}{llllllll}\mathrm{O} & 1 & 1.86037 & 2 & 180.0000 & 4 & 180.0000\end{array}$

$\begin{array}{lllllll}\mathrm{H} & 5 & 0.96434 & 1 & 127.0578 & 3 & -89.9738\end{array}$

$\begin{array}{lllllll}\text { H } & 5 & 0.96434 & 6 & 105.8842 & 1 & -180.0000\end{array}$

$\mathrm{Li}^{+}-\left(\mathrm{H}_{2} \mathrm{O}\right)_{3}$

(a B3LYP/6-311++g(3d,2p)

$\mathrm{Li}$

O $11 \quad 1.92654$

$\begin{array}{llllll}\mathrm{H} & 2 & 0.96722 & 1 & 127.5374\end{array}$ 


$\begin{array}{lllllll}\mathrm{H} & 2 & 0.96722 & 3 & 104.9252 & 1 & 180.0000 \\ \mathrm{O} & 1 & 1.92654 & 2 & 120.0000 & 3 & 36.6635 \\ \mathrm{O} & 1 & 1.92654 & 2 & 120.0000 & 5 & 180.0000 \\ \mathrm{H} & 5 & 0.96722 & 1 & 127.5374 & 2 & 36.6636 \\ \mathrm{H} & 5 & 0.96722 & 7 & 104.9252 & 1 & -180.0000 \\ \mathrm{H} & 6 & 0.96722 & 1 & 127.5374 & 5 & 36.6635 \\ \mathrm{H} & 6 & 0.96722 & 9 & 104.9252 & 1 & 180.0000\end{array}$
$\mathrm{Na}^{+}-\left(\mathrm{H}_{2} \mathrm{O}\right)_{2}$
@ B3LYP/6-311++g(3d,2p)

$\begin{array}{lllllll}\mathrm{Na} & & & & & \\ \mathrm{O} & 1 & 2.24530 & & & & \\ \mathrm{H} & 2 & 0.96366 & 1 & 127.4190 & \\ \mathrm{H} & 2 & 0.96366 & 3 & 105.1621 & 1 & -180.0000 \\ \mathrm{O} & 1 & 2.24530 & 2 & 180.0000 & 3 & -180.0000 \\ \mathrm{H} & 5 & 0.96366 & 1 & 127.4190 & 3 & -89.9970 \\ \mathrm{H} & 5 & 0.96366 & 6 & 105.1621 & 1 & -180.0000\end{array}$

\section{MODIFIED BASIS SET EXPONENTS}

\begin{tabular}{|c|c|c|c|}
\hline Atom & Function Type & Original exponent & Modified Exponent \\
\hline $\mathrm{O}$ in $\mathrm{Li}+\mathrm{H}_{2} \mathrm{O}$ & $\mathrm{F}$ & --- & 0.8203499 \\
\hline $\mathrm{Na}$ & $\mathrm{S}$ & 0.097000 & 0.0066500 \\
\hline & $\mathrm{P}$ & 0.1689 & 0.0070 \\
\hline $\mathrm{O}$ & $\mathrm{S}$ & 0.0737600 & 0.024586 \\
\hline & & & 0.098496 \\
\hline & & 0.0597400 & 0.052725 \\
\hline & $\mathrm{P}$ & & 0.430082 \\
\hline & & & 0.169341 \\
\hline & & --- & 0.098894 \\
\hline & $\mathrm{D}$ & & 0.622557 \\
\hline
\end{tabular}

In $\mathrm{Li}^{+}-\mathrm{H}_{2} \mathrm{O}$, an extra $\mathrm{F}$ type function is added on Oxygen.

For $\mathrm{Na}^{+}-\mathrm{H} 2 \mathrm{O}$, original $\mathrm{S}$ and P-type functions are replaced with the new modified $\mathrm{S}$ and $\mathrm{P}-$ type functions respectively. An extra $1 \mathrm{D}$ type function was added. 